\title{
Estrategias competitivas para los productores cafetaleros de la región de Córdoba, Veracruz, frente al mercado mundial
}

\author{
Joaquín Perea Quezada* \\ Luis Arturo Rivas Tovar**
}

\begin{abstract}
Resumen
Este trabajo tiene como objetivo identificar las estrategias competitivas para los productores cafetaleros de la región de Córdoba, Veracruz. Esto les permitirá competir en un mercado mundial globalizado, el cual se caracteriza por la sobreproducción de café en países como Brasil, Vietnam y Colombia, la fuerte competencia en el mercado de materias primas, el control del grano por un número limitado de países torrefactores y el descenso sostenido de los precios del café oro en el mercado mundial. Estas condiciones complicaron la atención de los cultivos para algunos productores y el abandono total de sus plantaciones para otros, como resultado del descenso de los precios internacionales. El descuido de la producción nacional y la pérdida de espacios han sido aprovechados por los países que vieron la crisis como una oportunidad para extender sus mercados sobre sus competidores más débiles.
\end{abstract}

Palabras clave: agricultura cafetalera, torrefacción, control de mercados, prueba de hipótesis, técnicas de cultivo.

Fecha de recepción: 15-08-2006

Fecha de aceptación: 05-07-2007

\footnotetext{
* Catedrático de la Universidad Paccioli de Córdoba, Veracruz. Correo electrónico: joaquinperea@yahoo.es

** Profesor y director del Centro de Investigación en Ciencias Administrativas de la ESCA-IPN y profesor invitado de la Universidad Politécnica de Madrid. Correo electrónico: larivas33@hotmail.com
}

No. 224, enero-abril 2008:9-33 


\title{
Competitive Strategies for Coffee Producers of Region Cordoba, Veracruz, in Front of the World-Wide Market
}

\begin{abstract}
The present work must like objective identify the competitive strategies for the coffee producers of the Region of Cordoba that allow them to compete in a globalization world-wide market characterized by the overproduction of farmers countries like Brazil, Vietnam, and Colombia, fort competition in the market of raw materials, control of the grain by a limited number of roaster countries and a progressive reduction of coffee gold prices in the world-wide market. These conditions complicate the attention of cultures of some producers and the total abandonment of plantations for others farmers like result of the reduction the international prices. The negligence of national production and loss of markets have been taken advantage by the countries that the crisis like an opportunity saw to extend their markets on their weaker competitors.
\end{abstract}

Key Words: Coffee agriculture, roasting, control of markets, test of hypothesis, techniques of culture.

\section{Introducción}

$\mathrm{C}^{1}$ café es una planta que posee el alcaloide de la cafeína, descubierto en 1895 por CFischer, aunque su efecto estimulante en el sistema nervioso fue descubierto hasta 1982. Esta planta se conoce por primera vez en Etiopía alrededor del siglo IX de nuestra era; sin embargo, es hasta el siglo XV cuando la semilla es plantada al sur de la península arábiga y se empieza a utilizar en infusiones de consumo popular. En el siglo XVII es llevada por holandeses a Europa y los franceses se encargan de transportarla a las Antillas, de donde finalmente se trae a México en 1790 (Uruchurtu, agosto 2004, p. 19), propiamente a la región de Córdoba, Veracruz; para 1847 esta planta ya se sembraba en los estados de Michoacán y Chiapas.

Su producción agrícola — que resulta ser fuente de trabajo, de ingresos y de divisas para el país- es un elemento de conservación de suelos y biodiversidad en los sistemas menos tecnificados; al mismo tiempo, fuente de conflictos, explotación y contaminación de ríos con la participación de diversos grupos étnicos y clases sociales, forzados por las políticas neoliberales a competir en el mercado mundial.

La producción mundial de este producto entre 2006 y 2007 fue de 128.5 millones de sacos de $60 \mathrm{~kg}$ en la que México ocupa el séptimo lugar (3.3\%). En primer lugar se encuentra Brasil (36.2\%), seguido por Vietnam (12.8\%), Colombia (9.5\%), Indonesia 
Estrategias competitivas para los productores cafetaleros

de la región de Córdoba, Veracruz, frente al mercado mundial

(6.1\%), Etiopía (4.3\%) e India (3.6\%) (www.fas.usda.gov/scriptsw). La excesiva producción de Vietnam y su propuesta de incrementar sus plantaciones de café arábica en un espacio no mayor a ocho años intensificó la competencia internacional deprimiendo los precios. Para la cosecha 2006-2007 Brasil aumentó su oferta mundial de café arábica en 10 millones 400 mil sacos de $60 \mathrm{~kg}$, lo que aceleró aún más su caída. La producción de México presenta una tendencia descendente en las últimas seis cosechas descuidando parte de sus mercados, que de inmediato han sido atendidos por Brasil y Colombia.

Los países cafetaleros se han ocupado de exportar en promedio el $80 \%$ de su producción de café verde permitiendo que países desarrollados como Estados Unidos, Alemania, Japón, Italia, Suiza, Bélgica, Holanda y Francia, entre otros, se ocupen de controlar e industrializar la mayor parte de la producción mundial del café (http://www.fas.usda. gov/scriptsw/attacherep/default.asp).

Del total de café verde exportado, México vende el 76.8\% a Estados Unidos, el 8.2\% a Suiza, el 3\% a Alemania, el 2\% a Francia y 2\% a Holanda. Por su parte, la demanda en México crece a una tasa media anual del $2.6 \%$ atendida con producción nacional y solubles importados de Estados Unidos y Canadá. Los solubles ${ }^{1}$ de importación están creciendo al $54 \%$ anual para cubrir la demanda promedio por habitante en el país de 734 gramos de café al año.

Los precios que se cotizan en el mercado internacional afectan a los productores que venden café cereza a precios que en la mayoría de los casos sólo les alcanza para pagar el corte. Asimismo, los pergamineros ${ }^{2}$ agregan valor a su producto, pero no logran recuperarlo en el mercado por deficiencias en la selección, calidad y falta de certificación.

El 88\% de la producción nacional se concentra en cuatro estados: Chiapas (35\%), Veracruz (25\%), Puebla (15\%) y Oaxaca (13\%) con los rendimientos más altos por hectárea en los primeros tres estados (Estadísticas COVERCAFÉ).

Del total de cultivos de café en el estado de Veracruz, el $72 \%$ se localiza en zonas con una altura superior a 600 metros sobre el nivel del mar donde se siembra café prima lavado, altura y estrictamente altura ${ }^{3}$ beneficiado en el mercado internacional con un sobre precio por su calidad, cuerpo, sabor y aroma (Estadísticas COVERCAFÉ). En cuanto a los 82 municipios del estado de Veracruz que cultivan café, 31 concentran

\footnotetext{
${ }^{1}$ Extracto de café vendido en polvo al consumidor

${ }^{2}$ Productores que después de despulpar el café cereza maduro lo secan con la cascarilla transparente que cubre el grano.

${ }^{3}$ La calidad del café se determina por su altura sobre el nivel del mar, a mayor altura mayor calidad (estrictamente altura). Los mejor lavados son los de inferior calidad.
} 
el $82 \%$ de la producción estatal con un promedio de 2.26 hectáreas por cada uno de los 67,579 productores. Por su parte, la región de Córdoba cuenta con 135 comunidades cafetaleras establecidas en 15 municipios con 12,460 productores que cultivan 28,514 hectáreas (Estadísticas COVERCAFÉ). El 83.3\% de la producción regional se concentra en 6 municipios donde se produce café prima lavado (34\%), altura y estrictamente altura (47.6\%).

La situación problemática que enfrentan los productores cafetaleros en el cultivo por los bajos precios del mercado se centra en: el descuido de sus plantaciones en la poda, control de malezas, fertilización, densidad de plantas y control de plagas que reducen su productividad por hectárea. Asimismo, el incumplimiento de las condiciones de certificación del café de especialidad ${ }^{4}$ mantiene a los productores al margen de su posible participación en los mercados mundiales de productos sustentables con la naturaleza.

Por otro lado, la falta de capacitación de productores y jornaleros en el manejo del café cereza $^{5}$, pergamino, verde ${ }^{6}$, tostado y molido dificulta su mayor participación en las cadenas de valor al desconocer el proceso que les permita conservar las propiedades del café y diferenciar su producto. Aunado a ello, su falta de organización dificulta el logro de apoyos financieros, apoyos en la comercialización y apoyos gubernamentales.

Los problemas cafetaleros más importantes se pueden resumir de la siguiente forma:

- La producción cafetalera nacional descendió de 6.2 millones de sacos de 60 kg. en la cosecha 1999-2000 a 3.9 millones para la cosecha 2003-2004 y 4.2 para la cosecha 2006-2007.

- Los mercados desatendidos por México fueron cubiertos por Brasil, Vietnam, Indonesia e India.

- Se vende café verde con escaso valor agregado en lugar del tostado, molido, soluble y descafeinado con mayor valor agregado.

- La demanda promedio por habitante en el país es ligeramente superior al medio kilo a diferencia del consumo de Brasil con $4.5 \mathrm{~kg}$. y Finlandia con $11.2 \mathrm{~kg}$.

- El 54.8\% de la producción mundial es industrializada por Estados Unidos, Alemania, Japón, Francia, Italia, España, Bélgica y Canadá principalmente para atender el mercado local y exterior.

\footnotetext{
${ }^{4}$ Cafés tratados desde el cultivo con insumos orgánicos o bajo sombra (gourmet) al margen del café convencional

${ }^{5}$ Pulpa madura cortada de la planta sin transformación

${ }^{6}$ Es el grano de café seco, libre de la pulpa y la cascarilla de color verde olivo que está listo para tostarse; también se le conoce como café oro
} 
Estrategias competitivas para los productores cafetaleros

de la región de Córdoba, Veracruz, frente al mercado mundial

- El mercado mexicano es atendido con el $75 \%$ de producción interna y el $25 \%$ con importaciones principalmente de Brasil.

- Los precios internacionales cayeron de 145.95 dólares el quintal ${ }^{7}$ en 1995 a 45.09 dólares en 2002 y repuntaron nuevamente hasta alcanzar 100.97 dólares el quintal en 2006.

- El sostenido descenso de los precios internacionales provocó el descuido de las plantaciones por los productores mexicanos que perdieron la oportunidad de aprovechar el repunte de precios generados por el descenso de la oferta en la cosecha 2005/2006.

- Las cosechas no se clasifican por calidad del grano ni se industrializan para alcanzar mejores precios.

- Los productores venden su cosecha en el mercado local principalmente en cereza.

- Hay desconfianza en la creación de sociedades de productores.

- Se carece de apoyos financieros. Se cuenta sólo con los subsidios de gobierno.

- Los cultivos con dos hectáreas en promedio por productor representan una importante fuente de ingresos para el productor cuando el precio de venta les permite cubrir sus costos. Al precio actual sólo pagan el corte.

- Se carece de mano de obra por la emigración de jornaleros y por los bajos salarios.

\section{Marco teórico}

Después de analizar las teorías propuestas por diferentes autores ${ }^{8}$ que explican las estrategias de diferenciación, se identificaron 65 variables, de las cuales siete se citan con mayor frecuencia:

- Capacidad de producción

- Innovación

- Calidad

- Mercadotecnia (a la que se integran variables como participación en el mercado, logística externa y lealtad a la marca por su escasa representatividad en el estudio)

- Organización

- Capacitación mano de obra

\footnotetext{
${ }^{7}$ Un quintal es igual a $240 \mathrm{~kg}$. de café cereza, $57.5 \mathrm{~kg}$. de café pergamino, $46 \mathrm{~kg}$. de café verde y $35 \mathrm{~kg}$. de tostado y molido. (La cotización en el mercado mundial se refiere al café verde de mayor comercialización). ${ }^{8}$ Gaither y Frazier (2000, pp. 230 y 630); Schroeder (1992, pp. 97-99); Harrison y St. John H. C. (2002 p. 116), Stanton Etzel y Walker (1996, pp. 7, 98, 467); Diez de Castro et al. (2001, pp. 67, 225, 349-351); Hill y Jones (1996, pp. 160-164, 176-182); Kotter y Schlesinger (1979, p. 57); Hodge, Anthony y Gales (1998, p.11); Mendoza (1998, pp. 61-81); Gitman (1986, p. 19); Sallenave (1994, pp.86-101); Perea (julio-diciembre, 2003, p. 35); Porter (1982, p. 16).
} 
- Rentabilidad, que incluye recursos financieros al no ser los productores sujetos de crédito

La definición de variables, sus dimensiones e indicadores de medición se describen a continuación.

\section{Capacidad de producción}

La capacidad de producción está determinada por el tamaño de la planta, por el número y habilidad de sus trabajadores, por el abasto de materiales, por la capacidad técnica del equipo y por la variedad de productos fabricados. La Federal Reserve Board (citada en Gaither y Frazier, 2000, p. 230) afirma:

La capacidad de producción es la tasa máxima de producción de una organización "nivel más elevado de volumen de producción que puede mantener una planta, dentro del marco de un programa realista de trabajo, tomando en cuenta el tiempo muerto normal y suponiendo una disponibilidad de insumos suficiente para operar la máquina y el equipo instalado."

La tasa máxima de producción estará limitada por las variaciones diarias de producción, el ausentismo de los empleados, vacaciones, retrasos en la entrega de materiales, mezcla de productos, nivel de la capacidad práctica y fallas en el equipo (Gaither y Frazier, 2000, p. 230).

\section{Innovación}

Para Schroeder (1992, pp. 98-99), a la innovación se le debe considerar como un impulso al mercado, fabricando lo que se puede vender; impulso a la tecnología, vendiendo lo que se puede hacer; e interfuncional, buscando la cooperación entre las diferentes áreas involucradas. Los pasos por seguir en un proceso de innovación se centran en la generación de la idea, selección del producto, diseño preliminar del prototipo, construcción del prototipo, pruebas y diseño definitivo del producto.

\section{Calidad}

Gaither y Frazier (2000, p. 630) afirman que la calidad de un producto o servicio es el grado de percepción del cliente en que dicho bien cumple con sus expectativas; asimismo, señalan que la calidad se determina por el desempeño del producto, características atractivas para el cliente, confiabilidad, capacidad de reparación, durabilidad, apariencia, seguridad y servicio. 
Estrategias competitivas para los productores cafetaleros

de la región de Córdoba, Veracruz, frente al mercado mundial

\section{Mercadotecnia}

Con relación a las estrategias de crecimiento:

[...] el marketing identifica nuevos clientes potenciales, propone oportunidades de productos, diseña campañas publicitarias y promocionales, organiza canales de distribución y crea políticas de precios y servicios de atención al cliente que ayudan a posicionar los productos de la compañía para los grupos de clientes adecuados. (Jeffrey, H. Caron J., 2002, p. 116)

\section{Participación en el mercado}

Para Stanton, Etzel y Walter (1996, p. 99) la participación en el mercado es la proporción de ventas totales de un producto durante determinado periodo en un mercado específico capturado por una compañía. De igual forma, también se puede considerar como la parte potencial del mercado que una compañía piensa alcanzar sobre el volumen de ventas que todas las empresas que venden un producto durante determinado periodo pueden esperar vender bajo condiciones ideales (Stanton, Etzel y Walter, 1996, p. 98).

\section{Logística externa}

Diez de Castro et al. (2001, p. 267) afirman que la logística externa está relacionada con las actividades de recepción del producto terminado, almacenamiento y distribución que consiste en hacer llegar el producto a su mercado meta. Es el proceso de transferir la propiedad del fabricante al consumidor final e incluir actividades como promover el producto, almacenarlo y correr parte del riesgo financiero durante el proceso de distribución. Las instituciones que pueden intervenir en el proceso de distribución son los bancos, las compañías de seguros, compañías de almacenamiento y transportistas.

\section{Lealtad a la marca}

La lealtad de los clientes está en función de la habilidad de la empresa para satisfacer sus necesidades; para lograrlo será necesario concentrarse con liderazgo en el cliente, estructurar actitudes del trabajador y mecanismos para llevar a los clientes a la organización, así como satisfacer sus necesidades a través de la personalización del servicio y tiempo de respuesta (Hill et al., 1996, pp. 160-164).

\section{Organización}

Es evidente la dificultad para convencer a la gente del cambio cuando se ha acostumbrado a cierta forma de vida que le permite resolver cómoda o incómodamente sus 
problemas. Todo cambio implica incertidumbre y pocas personas están dispuestas al riesgo aun con la promesa de cambios favorables. Para suavizar y vencer la resistencia al cambio en las organizaciones, Kotter y Schlesinger (1979, p. 57) proponen lo siguiente:

- Formación y compromiso. Compartir objetivos, conocimientos y percepciones con el personal que va a afectar el cambio.

- Participación e implicación. Las personas que participen deben ser implicadas desde la planeación y puesta en marcha.

- Apoyo y consejo. Proporcionar apoyo moral para reducir su ansiedad y sus miedos acerca del cambio

- Negociación y acuerdo. El compromiso mediante un intercambio de intereses mutuamente satisfechos es otra opción para reducir la resistencia al cambio.

- Manipulación y cooptación. Manipulación mediante promesas o perspectivas falsas.

- Coerción. Desechando cualquier propósito de consenso.

La intervención del Estado en la organización de cooperativas y el apoyo del partido oficial para fortalecer el corporativismo facilitó la integración campesina; sin embargo, la globalización y el retiro del gobierno deja al campesinado sin liderazgo y en el abandono con graves dificultades para volver a organizarse (Salminis, III cuatrimestre, 2003, p.77).

\section{Capacitación de la mano de obra}

Los procedimientos para la determinación de necesidades de capacitación Mendoza (1998, pp. 61-81) los clasifica en manifiestas y encubiertas. Las primeras son previsibles y se presentan cuando hay nuevos ingresos, promociones, transferencias, cambios de equipo y herramientas, cambios de procedimientos, de políticas, incrementos de estándares y metas más elevadas. Las necesidades de capacitación encubiertas o no previsibles presentan dificultades para su determinación como actitudes del personal, estilos de liderazgo, clima de la organización, factores motivacionales, costumbres, prejuicios y cultura de trabajadores y directivos.

\section{Recursos financieros}

Los recursos financieros tienen como función mantener la solvencia de la empresa en el suministro de flujos de efectivo necesarios para satisfacer las obligaciones y adquirir los activos fijos y circulantes necesarios para lograr los objetivos de la compañía (Gitman, 1986, p. 5). Para disponer de recursos financieros es necesaria la medición en las empresas del riesgo, rendimiento, valor del dinero en el tiempo, apalancamiento operativo y apalancamiento financiero (ibid., p. 19). 
Estrategias competitivas para los productores cafetaleros

de la región de Córdoba, Veracruz, frente al mercado mundial

\section{Rentabilidad}

Las empresas tienen muchos objetivos, pero para Sallenave (1994, pp. 86-101) se pueden reducir a tres: rentabilidad, crecimiento y supervivencia. Hasta los años setenta se ponía énfasis a la rentabilidad; en los setenta y ochenta se buscaba el crecimiento; actualmente, se busca la supervivencia porque ésta supone las anteriores. Sin rentabilidad y crecimiento no hay supervivencia.

Existen cuatro nociones fundamentales de rentabilidad: rentabilidad sobre ventas, rentabilidad económica, rentabilidad financiera (determinada por la rentabilidad del capital) y rentabilidad de un proyecto (Sallenave, 1994, p. 86). Esta última medida a través de índices dinámicos de desempeño futuro como el valor presente neto VPN que se define como el conjunto de flujos de caja actuales y futuros actualizados al costo de capital:

$$
V P N=\sum_{t=1}^{n} \frac{C F t}{(1+K o)^{t}}
$$

donde:

$C F_{t-}$ es el flujo de efectivo neto

${ }_{t}=$ es el horizonte de tiempo (años)

$K_{o}=$ es una tasa de actualización (se utiliza generalmente el costo de capital después de impuestos)

\section{Estrategias de diferenciación}

El objetivo de una estrategia genérica de diferenciación consiste en lograr una ventaja competitiva al crear un bien o servicio que sea percibido por los clientes como exclusivo de una manera importante; sin embargo, los clientes pagan un precio superior porque consideran que las cualidades diferenciales del producto valen la pena y porque el producto recibe un precio que se ha determinado en el mercado (Hofer y Schendel, 1978).

Para Mintzberg (1988, pp. 1-67) las estrategias de diferenciación se explican por el diseño (perfeccionar las características del producto y el diseño), la calidad (requiere de fiabilidad duración y funcionamiento del producto en relación con el precio), el suministro (promover un conjunto de productos básicos que satisfagan las necesidades de los consumidores dentro de un segmento) y la no diferenciación del producto (sin base para diferenciar, o seguir una estrategia de copiar lo ya existente). 
La diferenciación puede ofrecerse en la indiferenciación de productos debido a que el consumidor puede elegir entre varios oferentes atraído sólo por el servicio, como en el caso de algunas gasolineras que ofrecen valor agregado en su producto por el mismo precio (Samuelson y Nordhaus, 2006, p. 185).

En cambio para Dickson y Ginter (abril, 1987, pp. 1-10), la diferenciación está referida a la posición de la firma dentro del mercado o segmento del mercado con relación a su producto, servicio, imagen, características que influyen en el consumidor para su selección. La diferenciación puede ser tangible e intangible.

Autores como Thompson, Strickland y Gamble (2005, p.127) consideran que la diferenciación en los productos se puede lograr desde distintos ángulos: un sabor especial (unique taste), características múltiples, tiendas de descuento (one stop shopping), eficiencia en el servicio, disponibilidad de partes de repuesto, diseños de ingeniería y ejecución, prestigio y distinción, productos confiables, manufactura de calidad, tecnología de liderazgo, amplio rango de servicios, líneas completas de productos, productos exclusivos en imagen y reputación (top of the line).

\section{Metodología de la investigación}

\section{Objetivo general}

Con los antecedentes de la problemática cafetalera y el marco teórico descrito, se propone en la presente investigación identificar los factores que permitan a los productores cafetaleros de la región de Córdoba, Veracruz, lograr estrategias de diferenciación frente a los productores internacionales.

\section{Justificación}

El presente trabajo de investigación propone a los productores cafetaleros de la región de Córdoba, Veracruz, estrategias de diferenciación que les permitan hacer rentables sus cultivos, así como conservar el interés por conservarlos; además de sensibilizar la conciencia pública y privada sobre la importancia de la actividad agraria y la necesidad de conservarla próspera para el bien común. Asimismo, proponer estrategias de cambio en la conducta de los productores cafetaleros de la región de Córdoba para que acepten asociarse y adaptarse a los cambios que implica la globalización y libre mercado. De igual forma, despertar el interés en la realización de nuevas investigaciones en las distintas regiones del país que sustenten la metodología utilizada en la presente investigación y generen aportaciones nuevas en un sector de importancia vital y sustentable para la nación como es el sector agropecuario. 
Estrategias competitivas para los productores cafetaleros

de la región de Córdoba, Veracruz, frente al mercado mundial

\section{Problema de investigación}

Se desconoce si las variables definidas en el marco teórico son determinantes para que los productores cafetaleros cuenten con estrategias de diferenciación, y si al manipularse cada una de ellas resuelvan el problema de dependencia de los precios externos; sin embargo, se han definido como propuesta y se espera probarlo a través del proceso de investigación.

\section{Preguntas de investigación}

1. ¿La capacidad de producción de los cafetaleros de la región de Córdoba, Veracruz, contribuye a la creación de estrategias de diferenciación?

2. ¿La innovación en los procesos que realizan los productores cafetaleros de la región de Córdoba, Veracruz, es determinante de las estrategias de diferenciación?

3. ¿El descuido en la calidad de los procesos realizados por los productores cafetaleros de la región de Córdoba, Veracruz, es causal de las estrategias de diferenciación?

4. ¿La falta de atención a las actividades de mercadotecnia por los productores cafetaleros de la región de Córdoba, Veracruz, genera ausencia de estrategias de diferenciación?

5. ¿La falta de organización de los productores cafetaleros de la región de Córdoba, Veracruz, dificulta la presencia de estrategias de diferenciación que les permita defender sus intereses?

6. ¿La capacitación de la mano de obra cafetalera de la región de Córdoba, Veracruz, generaría estrategias de diferenciación?

7. ¿Si los productores cafetaleros de la región de Córdoba, Veracruz, cuentan con recursos financieros podrán crear estrategias de diferenciación que les permitan sobrevivir con sus cultivos?

8. ¿Si los productores cafetaleros de la región de Córdoba, Veracruz, realizan actividades que generen rentabilidad en sus cultivos contribuirán a la creación de estrategias de diferenciación?

Para la presente investigación —-tomando en cuenta que ya se han realizado investigaciones correlacionales previas como las propuestas por Bonales (2001) y Chauca (2000), quienes han probado la validez y confiabilidad de las variables seleccionadas- se intentará establecer el método de análisis causal multivariado entre las variables independientes definidas en el marco teórico. 


\section{Hipótesis general}

La hipótesis que se describe a continuación surge como resultado del análisis del marco teórico y del análisis documental cafetalero con un sentido de causa efecto para tratar de dar respuesta a los problemas que enfrentan los productores y establecer las estrategias por seguir para mantenerse en el mercado.

Las estrategias de diferenciación en la producción cafetalera de la región de Córdoba, Veracruz, se explican por la capacidad de producción, innovación, calidad, mercadotecnia, participación en el mercado, logística externa, lealtad a la marca, organización, capacitación de la mano de obra, recursos financieros y rentabilidad.

\section{Horizonte temporal y espacial}

El trabajo de investigación se realizó con información obtenida de los productores cafetaleros de los municipios de Córdoba, Fortín, Ixtaczoquitlán, Chocamán, Amatlán de los Reyes, Ixhuatlán del Café, Tepatlaxco, Naranjal y Atoyac, pertenecientes a la región de Córdoba, durante la cosecha 2003-2004.

\section{Universo}

De los quince municipios que integran la región de Córdoba, en nueve se produce el $96.7 \%$ de la cosecha cafetalera con un total con 10,996 productores. Por el tamaño del predio, $53.6 \%$ de los cafetaleros no poseen más de tres hectáreas, el $28.5 \%$ poseen más de tres y hasta siete, el $12.8 \%$ poseen más de siete y hasta veinte, el $5.1 \%$ posee más de veinte y hasta 250 hectáreas. Por el tipo de café que se produce de acuerdo con la altura sobre el nivel del mar, los productores obtienen $27.9 \%$ de café estrictamente altura, $24.5 \%$ café de altura, $31.4 \%$ café prima lavado y el $16.2 \%$ café buen lavado. La muestra por seleccionar debe ser representativa de todos los municipios, productores, tamaño de predios y tipos de cultivo, según su altura sobre el nivel del mar (véase cuadro 1).

\section{Tamaño de la muestra}

El método utilizado para calcular el tamaño de la muestra se describe a continuación (Hernández et al., 2003, pp. 309-310).

$$
\begin{aligned}
& \mathrm{n}^{\prime}=\left(\mathrm{S}^{2} / \mathrm{V}^{2}\right) \\
& \mathrm{S}^{2}=\mathrm{p}(1-\mathrm{p})
\end{aligned}
$$


Estrategias competitivas para los productores cafetaleros

de la región de Córdoba, Veracruz, frente al mercado mundial

donde:

$\mathrm{p}=$ margen de error aceptado

$\mathrm{V}=$ error estándar de la población

$\mathrm{n}=\mathrm{n}^{\prime} /\left(1+\left(\mathrm{n}^{\prime} / \mathrm{N}\right)\right)$

$\mathrm{N}=$ tamaño de la población

$\mathrm{S}=$ Desviación estándar de la muestra expresada como probabilidad de ocurrencia de y

$\mathrm{y}=$ Valor promedio de la variable $=1$

$\mathrm{S}^{2}=$ varianza de la muestra expresada como la probabilidad de ocurrencia de y

Si se calcula la muestra con los siguientes datos:

$\mathrm{N}=10996$ productores cafetaleros

$\mathrm{p}=$ margen de error .10

$\mathrm{V}=.02474$

$\mathrm{S}^{2}=(.10(1-.10))=.09$

$\mathrm{V}^{2}=(.02474)^{2}=.000612245$

$\mathrm{n}^{\prime}=.(.09 / .000612245)=147$

$\mathrm{n}=147 /(1+(147 / 10996))=\mathbf{1 4 5}$

El tamaño de la muestra se estima en 145 productores.

\section{Sujetos de investigación}

La población o universo integrado por 10,996 productores, después de la conversión de los datos originales en su estructura porcentual y convertida a números absolutos generó los resultados que aparecen en el cuadro 1.

Referente al tamaño del predio, 58 productores corresponden a los que tienen máximo una hectárea, 65 cuentan con más de una y no más de tres, 14 poseen más de tres y al menos cinco, 8 son dueños de predios con más de cinco hectáreas y al menos 20. Por su altura sobre el nivel del mar, forman parte de la muestra 41 productores que producen café estrictamente altura, 40 café de altura, 52 prima lavado y 12 café buen lavado.

\section{Instrumento de medición}

Definido el universo de 10,996 productores, el horizonte espacial que comprende la región de Córdoba y el horizonte temporal para la aplicación de cuestionarios durante la cosecha 2003-2004 fue posible definir la muestra y los sujetos por entrevistar.

Con base en la estratificación de productores por municipio se calculó la muestra para entrevistar a 145 productores cafetaleros a quienes se les aplicó personalmente, y en sus 
domicilios, un cuestionario de tipo cuantitativo de 44 ítems, cabe aclarar que originalmente constaba de 101 preguntas.

En la información de cada ítem se utilizaron escalas de medición nominal, ordinal, por intervalos y de razón.

Cuadro 1

Muestra poblacional por tamaño de predio en hectáreas por productor

\begin{tabular}{|c|c|c|c|c|c|c|c|c|}
\hline Municipios & Total & $0<\mathrm{X} \leq 1$ & $1<\mathrm{X} \leq 3$ & $3<\mathrm{X} \leq 5$ & $5<\mathrm{X} \leq 7$ & $7<\mathrm{X} \leq 10$ & $10<\mathrm{X} \leq 15$ & $15<\mathrm{X}$ \\
\hline $\begin{array}{c}\text { estrictamente } \\
\text { altura }\end{array}$ & & & & & & & & \\
\hline Chocamán & 10 & 6 & 4 & 0 & 0 & 0 & 0 & 0 \\
\hline Ixhuatlán del Café & 31 & 10 & 13 & 4 & 1 & 1 & 1 & 1 \\
\hline Altura & & & & & & & & 0 \\
\hline Fortín & 11 & 5 & 5 & 1 & 0 & 0 & 0 & 0 \\
\hline Ixtaczoquitlán & 29 & 14 & 12 & 2 & 1 & 0 & 0 & 0 \\
\hline prima lavado & & & & & & & & 0 \\
\hline $\begin{array}{c}\text { Amatlán de los } \\
\text { Reyes }\end{array}$ & 13 & 4 & 6 & 2 & 1 & 0 & 0 & 0 \\
\hline Córdoba & 24 & 10 & 11 & 2 & 0 & 0 & 0 & 1 \\
\hline Naranjal & 5 & 2 & 2 & 0 & 0 & 0 & 0 & 1 \\
\hline Tepatlaxco & 10 & 3 & 6 & 1 & 0 & 0 & 0 & 0 \\
\hline buen lavado & & & & & & & & 0 \\
\hline Atoyac & 12 & 4 & 6 & 2 & 0 & 0 & 0 & 0 \\
\hline TOTAL & 145 & 58 & 65 & 14 & 3 & 1 & & 0 \\
\hline
\end{tabular}

Fuente: Estadísticas de covercafé con información del INMECAFÉ, México, 2001

El método de escalamiento tipo Likert (1976, citado en Summers, pp. 182-193) consiste en un conjunto de ítems presentados en forma de afirmaciones o juicios ante los cuales los sujetos eligen sólo uno de los cinco puntos de la escala; a cada punto se le asigna un valor numérico, con esto el sujeto obtiene una puntuación total sumando los resultados obtenidos en relación con todas las afirmaciones (véase cuadro 2).

Cuadro 2

Ejemplo de respuestas de ítems

\begin{tabular}{|c|c|c|c|c|c|}
\hline Puntaje & $\mathbf{5}$ & $\mathbf{4}$ & $\mathbf{3}$ & $\mathbf{2}$ & $\mathbf{1}$ \\
\hline Respuesta & $\begin{array}{c}\text { Muy de } \\
\text { acuerdo }\end{array}$ & De acuerdo & $\begin{array}{c}\text { Ni de acuerdo ni en } \\
\text { desacuerdo }\end{array}$ & $\begin{array}{c}\text { En } \\
\text { desacuerdo }\end{array}$ & $\begin{array}{c}\text { Muy en } \\
\text { desacuerdo }\end{array}$ \\
\hline
\end{tabular}

Fuente: Likert, 1976, citado en Summers, pp. 182-193

\section{Medición de la validez}

Un instrumento de medición debe cumplir con dos requisitos: validez y confiabilidad. La validez se refiere al grado en que un instrumento mide realmente lo que se preten- 
Estrategias competitivas para los productores cafetaleros

de la región de Córdoba, Veracruz, frente al mercado mundial

de medir y las "evidencias que se presenten deben estar relacionadas con el contenido; es decir, la medición debe representar el concepto efectivamente medido al compararlo con un criterio externo estándar (constructo) bajo el cual se juzga" (Wiersma, 1999, citado en Hernández et al., 2003, p. 347). La validez del constructo establece y especifica la relación entre el marco teórico y la variable definida; además, correlaciona y analiza cuidadosamente su relación e interpreta la evidencia empírica de acuerdo con el nivel en el que clarifica la validez de su medición. (Carmines y Zeller, 1988, Series Quantitative Applications in the social Sciences, vol. 17).

La validez del constructo se realizó con el procesamiento y análisis de datos de los ítems que integran cada variable del modelo, mediante el análisis de factor con el apoyo del paquete estadístico SPSS versión 12 para las ciencias sociales.

\section{Medición de la confiabilidad}

La confiabilidad se puede medir al aplicar un instrumento de medición dos o más veces a un mismo grupo de personas o aplicar dos o más instrumentos de medición a un mismo grupo en diferentes momentos. El método de mitades partidas requiere sólo una aplicación de la medición y compara puntuaciones de ambas partes que deben estar muy correlacionadas. El coeficiente Alfa de Cronbach que requiere sólo una administración del instrumento de medición para toda la población sin necesidad de dividirla.

El método utilizado, por las características de la investigación, fue el Coeficiente Alfa de Cronbach que requiere sólo una administración del instrumento de medición a toda la población cafetalera.

\section{Cuestionario}

De acuerdo con la técnica de escalamiento propuesta por Likert, el cuestionario definido se aplicó a 145 productores cafetaleros de la región de Córdoba con preguntas accesibles e interesantes para el productor. A los ítems elaborados se les asignaron siete probables respuestas de las cuales el productor sólo podía contestar una; en los casos en que había más de una respuesta se optó por la de mayor porcentaje. A cada respuesta se le asignó un valor numérico máximo de (7) si la respuesta representaba fuerte impacto en las estrategias de diferenciación y un valor numérico mínimo de (1) si su impacto era mínimo o nulo. 


\section{Resultados}

La puntuación máxima por pregunta (7) multiplicada por el número de ítems (44) por el número de productores encuestados (145) puede alcanzar un máximo de 44,660 puntos $(145)(7)(44)=44,660$; de lograrse se podría afirmar que los productores poseen estrategias de diferenciación a la altura de la competencia local y mundial. Sin embargo, los resultados generaron un puntaje de 25,947 (145 x 4.067 x $44=25,947)$ equivalentes al $58 \%$ del resultado esperado.

\section{Resultados de la aplicación de cuestionarios}

Los resultados por variable presentan a la calidad con el lugar más alto al afirmar los productores que no tienen reclamaciones en su producto y que tradicionalmente es bien aceptado por el comprador al cumplir con las expectativas del mercado. Es conveniente señalar que el $65 \%$ de los productores encuestados venden café cereza al momento de la cosecha a los acaparadores, quienes no exigen diferenciación ni calidad y pagan a todos el grano al mismo precio. La finalidad de los intermediarios (acaparadores) es captar la mayor cantidad de grano, ya que su comisión depende del volumen.

El segundo orden lo ocupa la variable rentabilidad donde el $79 \%$ de los productores afirma que el café es una fuente de recursos, avalado por el repunte del precio; el 91\% de ellos no están dispuestos a abandonar sus cultivos, pues esperan que la actitud de las autoridades y el mercado cambien en cualquier momento, como ha ocurrido en otras épocas.

Cuadro 3

Capacidad competitiva por variables

\begin{tabular}{|c|c|c|c|}
\hline Variables & $\begin{array}{c}\text { Puntaje } \\
\text { alcanzado }\end{array}$ & $\begin{array}{c}\text { Puntaje } \\
\text { esperado }\end{array}$ & $\begin{array}{c}\text { Índice de } \\
\text { competitividad }\end{array}$ \\
\hline $\begin{array}{c}\text { Capacidad de producción } \\
\text { Innovación } \\
\text { Calidad } \\
\text { Mercadotecnia } \\
\text { Organización } \\
\text { Capacitación mano obra } \\
\text { Rentabilidad }{ }^{2} \\
\text { Estrategias de diferenciación } \\
\text { Total }\end{array}$ & $\begin{array}{c}5396 \\
2340 \\
2493 \\
4734 \\
2389 \\
2378 \\
3085 \\
3132 \\
25947\end{array}$ & $\begin{array}{l}9135 \\
5075 \\
3045 \\
9135 \\
4060 \\
5075 \\
4060 \\
5075 \\
44660\end{array}$ & $\begin{array}{l}.591 \\
.461 \\
.818 \\
.518 \\
.588 \\
.469 \\
.760 \\
.617 \\
.581\end{array}$ \\
\hline
\end{tabular}

${ }^{1}$ Incluye los ítems de las variables lealtad a la marca, logística de salida, mercadotecnia y participación en el mercado ${ }^{2}$ Incluye los ítems de la variable recursos financieros y rentabilidad Fuente: Elaboración propia con datos del trabajo de campo 
Estrategias competitivas para los productores cafetaleros

de la región de Córdoba, Veracruz, frente al mercado mundial

El tercer lugar lo ocupa la variable dependiente estrategias de diferenciación, influida por la tendencia de respuestas a algunos ítems que presentan optimismo dentro de la desorganización y el atraso en la integración de cadenas de valor.

Los datos obtenidos en el trabajo de campo se procesaron utilizando los programas: SPSS 12.0 para Windows, Microsoft Word y Excel que permitieran probar el grado de dependencia entre las variables independientes y la variable dependiente.

Ed. $=b_{1}+b_{2}(C p)+b_{3}(M k)+b_{4}($ Inv $)+b_{5}($ Org $)+b_{6}\left(C_{m o}\right)+b_{7}(C a l)+.b_{8}($ Rnt $)+E$

Cada una de las variables se define como sigue:

Dependiente:

$\mathrm{Ed}=$ Estrategias de diferenciación

Independientes:

$\mathrm{b}_{0}=$ Parámetro que mide a la variable dependiente cuando las variables independientes carecen de cambios o su cambio es cero (0)

$b_{i}=$ Coeficiente que mide el impacto en la variable dependiente cuando la variable independiente cambia en una unidad.

$\mathrm{Cp}=$ Capacidad de producción.

$\mathrm{Mk}=$ Marketing

Inv $=$ Innovación

Org = Organización de productores.

$\mathrm{Cmo}=$ Capacitación de la mano de obra

$\mathrm{Cal}=$ Calidad

Rnt $=$ Rentabilidad

$\mathrm{E}=$ error en los datos, no explicado por la ecuación

Los resultados fueron como sigue:

Ed. $=-5.911-.104(\mathrm{Cp})+.276(\mathrm{Mk})+.193(\mathrm{Inv})+.164(\mathrm{Org})+.07639(\mathrm{Cmo})+.581(\mathrm{Cal})+$ .251 (Rnt)

$\mathrm{R}=.858$

$\mathrm{R}^{2}=.735$

$\mathrm{F}=54.4$

La correlación entre las variables independientes y la variable dependiente con un índice $\mathrm{R}=.858$ se considera marcada alta. 
Los coeficientes de cada variable independiente indican la magnitud del impacto que tiene cada una de ellas en la variable dependiente. Incrementar una unidad en la variable calidad constituye un incremento en la variable estrategias de diferenciación de .581. De la misma forma, el incremento en una unidad en la variable marketing tendrá un impacto de .276 en las estrategias de diferenciación.

El impacto de las variables independientes en la variable dependiente definida en conjunto para todos los productores encuestados generó los resultados y las ecuaciones por municipio que aparecen en el cuadro 4.

Cuadro 4

Impacto municipal de las variables independientes en la variable dependiente

\begin{tabular}{|c|c|c|c|c|c|c|c|c|c|c|c|c|}
\hline \multirow[b]{2}{*}{ Municipio } & \multicolumn{3}{|c|}{$\begin{array}{c}\text { Coeficientes } \\
\text { de correlación }\end{array}$} & \multicolumn{8}{|c|}{ Coeficientes de las variables independientes } & \multirow{2}{*}{$\begin{array}{l}\text { V.D. } \\
\text { E.Dif } \\
\text { E.D. }\end{array}$} \\
\hline & $\mathrm{R}$ & $\mathrm{R}^{2}$ & $\mathrm{~F}$ & $\begin{array}{l}\text { Const } \\
\mathrm{B}_{1 \mathrm{j}}\end{array}$ & $\begin{array}{l}\text { (Cp) } \\
b_{2 j}\end{array}$ & $\begin{array}{l}(\mathrm{Mk})^{*} \\
\mathrm{~b}_{3 \mathrm{j}}\end{array}$ & $\begin{array}{l}\text { (Inv) } \\
\mathrm{b}_{4 \mathrm{j}}\end{array}$ & $\begin{array}{l}(\text { Org) } \\
b_{5 j}\end{array}$ & $\begin{array}{l}\left(\mathrm{C}_{\mathrm{mo}}\right) \\
\mathrm{b}_{6 \mathrm{j}}\end{array}$ & $\begin{array}{l}(\mathrm{Cal}) \\
\mathrm{b}_{6 \mathrm{j}}\end{array}$ & $\begin{array}{l}\text { (Rnt)** } \\
b_{6 j}\end{array}$ & \\
\hline Amatlán & .893 & .798 & 2.8 & 7.719 & .393 & .133 & -.020 & -.405 & -.309 & .654 & -.435 & E.D \\
\hline Córdoba & .891 & .801 & 10.3 & 4.207 & .084 & -.206 & .050 & .484 & .312 & .303 & .057 & E. $D_{2}$ \\
\hline Fortín & .925 & .855 & 4.2 & 29.432 & -.047 & -.322 & .434 & -.200 & -.036 & .522 & -.355 & E.D. ${ }_{34}$ \\
\hline Ixhuatlán & .951 & .904 & 17.4 & -16.58 & -.022 & .457 & .337 & .088 & .076 & .855 & .008 & E.D. ${ }_{4}$ \\
\hline $\begin{array}{c}\text { Ixtaczoqui- } \\
\text { tlán }\end{array}$ & .885 & .783 & 12.3 & -11.48 & .042 & .231 & .130 & -.037 & .133 & .980 & .113 & E.D $D_{5}$ \\
\hline Tepatlaxco & .915 & .829 & 4.1 & -15.94 & -.111 & -.174 & -.001 & .308 & .013 & 2.248 & .180 & E.D. ${ }_{6}$ \\
\hline $\begin{array}{c}\text { Atoy_Choc- } \\
\text { Nar }\end{array}$ & .918 & .843 & 8.4 & -4.20 & -.134 & .275 & .234 & .156 & -.021 & .270 & .528 & E.D..$_{7}$ \\
\hline Acumulado & .858 & .735 & 54.4 & -5.91 & -.104 & .276 & .193 & .164 & .076 & .581 & .251 & $\mathrm{ED}_{8}$ \\
\hline
\end{tabular}

*Incluye variables: lealtad a la marca, logística de salida, mercadotecnia, participación de mercado ** Incluye variables: recursos financieros y rentabilidad

Fuente: Elaboración propia con los resultados del trabajo de campo

Los coeficientes de correlación presentan índices con calificación marcada alta, sin embargo, el análisis de varianza $\mathrm{F}$ dependiendo del número de cuestionarios aplicados por municipio dificultan aplicar con éxito esta prueba.

\section{Análisis}

La correlación entre el acumulado de variables con la variable dependiente estrategias de diferenciación (según Guilford, 1954) es marcada alta de .832. El resto de variables independientes con el acumulado están correlacionadas dentro de la categoría moderada sustancial, excepto innovación con .816, de acuerdo con las escalas establecidas en los coeficientes de correlación producto-momento de Pearson ( $\mathrm{Pa}-$ dua, 1996, pp. 286-287). 
Estrategias competitivas para los productores cafetaleros

de la región de Córdoba, Veracruz, frente al mercado mundial

El análisis de varianzas generó una $\mathrm{F}$ de 54.4 veces los errores, superior al número de veces esperado en tablas generando la aceptación de Ho.

El procedimiento definido por Carmines y Zeller (1988, pp. 44-45) para calcular el coeficiente $\alpha$ de Cronbach generó los siguientes resultados:

$\alpha=[\mathrm{NP}] /[1+(\mathrm{P}(\mathrm{N}-1)]$

$\alpha=$ Coeficiente de Confiabilidad Alfa de Cronbach

$\mathrm{N}=$ Número de ítems

$\mathrm{P}=$ Promedio de las correlaciones entre ítems

El coeficiente de Alfa Crombach para 44 ítems que comprenden las variables independientes y dependiente más el acumulado es como sigue:

$\alpha=[\mathrm{NP}] /[1+(\mathrm{P}(\mathrm{N}-1)]=44(.46119) /[1+.46119(44-1)]=.9643$

\section{Discusión}

\section{Sobre el marco teórico}

Por una parte, el estudio prueba que la agricultura sigue siendo un factor determinante de la riqueza para las empresas más grandes del mundo. Por otra, el control del tipo de cambio mantiene en desventaja a los productores cafetaleros nacionales frente a los productores internacionales que pueden vender sus productos en el país a precios más bajos.

\section{Sobre el trabajo de campo}

La capacidad competitiva de los productores cafetaleros fue del 58\% impulsada a la baja principalmente por la ausencia de abonos en sus cultivos. En cuanto a los ítems que presentan los índices de competitividad más bajos con respecto al $100 \%$ son los siguientes: aplicación de abonos (34.5\%), aplicación de plaguicidas $(20.5 \%)$, clasifican su café al cosecharlo (22.5\%), cuentan con capacitación en técnicas de comercialización (32.4\%), venden su producción en el mercado nacional (22.2\%), venden su producto en el mercado extranjero (14.3\%), realizan promoción o publicidad de su producto $(17 \%)$, competitividad de la venta de su producto en la cadena de valor $(30.1 \%)$. 


\section{Evaluación de resultados}

En el modelo se incluyeron variables como lealtad a la marca, logística externa, participación en el mercado que por su escasa o nula importancia en la actividad de los productores cafetaleros fue necesario incluirlas en la variable marketing. El mismo caso presenta la variable recursos financieros incluida en la variable rentabilidad ante la ausencia de ganancias en la actividad cafetalera que aleja a los banqueros privados y públicos de negocios no rentables.

La validez del modelo se genera a partir de la congruencia entre el problema de investigación, el objetivo, las preguntas de investigación, las hipótesis, la definición operacional de cada variable y la construcción de ítems que dan respuesta a las preguntas de investigación.

Por lo que respecta a la correlación entre variables, ésta se prueba a través de los coeficientes de correlación calculados entre las variables independientes y la variable dependiente.

\section{Propuestas}

Se prueba que las estrategias por parte de los productores para mantener competitivos sus cultivos no existen, que es necesario hacer tareas desde el cultivo que no se están realizando y que el apoyo del gobierno debe ser determinante y debe vincular los apoyos a la creación de valor en el café y no como un regalo a cambio del voto.

En relación con el exterior, es importante considerar lo siguiente:

1. Vincular la actividad industrial y de servicios con la actividad agrícola y, sobre todo, con la actividad cafetalera para beneficio de los participantes.

2. Evitar el subsidio que tiene más carácter político partidista que auténtica ayuda, al apoyar a productores que han dejado de cultivar café o premiar a los que conservan cultivos de café mal atendidos o abandonados.

3. Impulsar la producción nacional para cumplir con el mercado interno y marginalmente con el mercado exterior debido a que la escasez estimula las importaciones.

4. Atender el mercado interno con la producción nacional, impulsando la formación de cadenas de valor entre los participantes hacia el cliente.

5. Evitar en lo posible la venta de café verde en el mercado mundial controlado por los torrefactores ${ }^{9}$ extranjeros, al ser el más barato y con tendencia a la baja.

\footnotetext{
${ }^{9}$ Empresas extranjeras como Nestlé que concentran gran cantidad de grano mundial, lo industrializan y lo venden con un alto grado de diferenciación al consumidor. 
Estrategias competitivas para los productores cafetaleros

de la región de Córdoba, Veracruz, frente al mercado mundial

6. Aumentar la venta de tostados y solubles con mayor valor agregado, en el mercado mundial que cuenta con márgenes más amplios de ganancias.

7. Integrar con productores cadenas de valor que fomenten la venta directa al consumidor de café tostado y molido, soluble o descafeinado.

8. Estimular con apoyos de gobierno la atención de los cultivos por los productores cuando alcancen una productividad superior a tres toneladas de café cereza por hectárea. No apoyar tierras que se asume son cafetaleras, pues algunos productores reciben el apoyo y ya no son cafetaleros.

9. Los apoyos de gobierno deben ser orientados a la productividad y no a la posesión de tierras cafetaleras o al volumen de ventas de café cereza. Deben apoyar a los productores agrupados en la industrialización y comercialización de café convencional y orgánico que estimule la formación de cadenas de valor.

10. Integrar sociedades de productores que fomenten la formación de cadenas de valor desde el cultivo, proceso y comercialización del producto terminado con destino al consumidor, con un reparto equitativo de los beneficios en todas las etapas.

11. Es necesario impulsar la diversificación de productos hacia el consumidor con mayor margen de ganancia, suficiente para hacer atractivos los cultivos y la conservación de las plantaciones.

12. Se debe conservar una disciplina en la producción que evite escasez o sobre oferta que únicamente favorece a las empresas torrefactoras que controlan el mercado.

En relación con el trabajo de campo, es conveniente considerar lo siguiente:

1. Es necesario que el productor mejore sus cultivos, el $73.1 \%$ no los abona, el $92.5 \%$ no los protege contra las plagas y el $42.8 \%$ cuenta con plantíos entre 16 y 40 años de edad.

2. Se debe organizar a los productores para el tratamiento de su café. El proceso de innovación en los cultivos cafetaleros no requieren grandes descubrimientos, pero los indispensables no se hacen: el $82.6 \%$ vende su café cereza sin clasificarlo, revuelto y defectuoso; el $68.2 \%$ no dispone del equipo adecuado para mejorar el proceso de su café; el 50.4\% afirma no poder conseguirlo fácilmente por falta de dinero.

3. No existe lealtad del cliente al productor; no obstante que el $87.6 \%$ afirma que produce pensando en el cliente y el $98 \%$ señaló no tener devoluciones de los compradores, el $65 \%$ de los productores vende su café en cereza y el $57.9 \%$ vende su café a las compras en el campo, que por falta de valor agregado generalmente no tiene reclamaciones o devoluciones.

4. Al no industrializarse el producto, el $96 \%$ no promociona su producto y sólo el $13.8 \%$ ha logrado incrementar su producción y ventas.

5. Promover la venta del café en el mercado extranjero industrializado en descafeinados, solubles, tostados y molidos en lugar de pergaminos. El $65 \%$ vende 
su café en cereza en el campo, el 27.6\% lo vende en el mercado local, el 5.5\% en el mercado nacional y ningún productor de los entrevistados vende su producto en el mercado extranjero.

6. Proponer procesos para el café en los que participe el productor, puesto que el $32.4 \%$ afirma que tal vez sí maneje adecuadamente el producto, el $44.1 \%$ que muy probablemente sí y el 20.0\% definitivamente sí; sin embargo, sólo el 17.4\% que industrializa su café se compromete con el manejo del café después del corte; en cambio el $65 \%$ que lo vende en cereza no asume ningún compromiso.

7. Impulsar la diferenciación del producto debido a que el $21.4 \%$ de productores de todos los municipios afirma que tal vez sí se identifica el comprador con su producto, el $50.3 \%$ cree que muy probablemente sí y el $20.7 \%$ que definitivamente sí; sin embargo, si el $65 \%$ vende su café en cereza al momento de cosecha, el producto carece de valor agregado y al comprador no le importa la calidad ni quién es el vendedor.

8. Capacitar a los productores para que se organicen, pues el 58\% afirmó no pertenecer a organización alguna, el $60.7 \%$ carece de capacitación para integrarse a alguna, el $54.4 \%$ encuentra dificultades para trabajar en equipo, no obstante que el $86.9 \%$ supone que el organizarse le atraerá beneficios.

9. Capacitar a los productores porque el $62.1 \%$ desconoce las normas para producir buen café, el $62.8 \%$ desconoce los estándares que debe cumplir su producto cuando lo vende. Cabe aclarar que el 51.3\% carece de capacitación en técnicas de cultivo, el $68.2 \%$ desconoce las técnicas de proceso y el $88.3 \%$ desconoce las técnicas de comercialización.

10. Impulsar con apoyos de gobierno los cultivos que prometan ser rentables. Es necesario que los productores cafetaleros que reciben apoyos de gobierno $(85.5 \%$ de los encuestados) realmente los utilicen productivamente para que sean rentables y sujetos de crédito bancario público o privado.

11. Mantener vivo el interés de los productores de conservar sus cultivos, pues el $40.7 \%$ recibe a lo más dos pesos por kilo de café cereza y el $82.8 \%$ no recibe más de tres pesos por kilo aun si lo entrega procesado. El 27.6\% de productores supone que con un precio de venta de 4 pesos sería suficiente para atender sus cultivos, el $37.5 \%$ cree que serían suficientes 5 pesos y el $24 \%$ afirma que el precio del café cereza debe estar entre 3 y 3.5 pesos; en tanto que el $79.4 \%$ afirma que sus cultivos son rentables y, por lo tanto, el 97.9\% de los productores están dispuestos a conservar sus cultivos.

12. Cumplir desde el cultivo con tareas que no se están realizando y que el apoyo del gobierno se otorgue a condición de que se vinculen los apoyos a la creación de cadenas de valor hacia el cliente.

13. Promover la conversión de cultivos de café convencional a café orgánico con beneficios marginales en el precio y en el cuidado del medioambiente. 
Estrategias competitivas para los productores cafetaleros

de la región de Córdoba, Veracruz, frente al mercado mundial

14. Proponer la integración de organizaciones en las que participen en colaboración empresas de beneficio húmedo, beneficio seco y productores en un proceso de mejora continua asumiendo el compromiso de calidad con el consumidor.

15. Colaborar con los intermediarios locales de beneficio húmedo y seco e integrarlos a la cadena de valor, compartiendo el proceso de camino al cliente frente a los torrefactores internacionales que operan en el país.

16. Realizar el proceso de integración vertical logrando alianzas con los participantes y el compromiso de agregar valor a la cadena de un producto que es atractivo para el consumidor, ampliamente diferenciable y que garantiza a los participantes seguridad y permanencia en el futuro. Un reto en el proceso de integración para los productores es el número de participantes debido a que la mayoría cuenta como máximo con dos hectáreas de tierra en promedio.

\section{Bibliografía}

Bonales, V. (2001), Competitividad de las empresas de Uruapan, Michoacán, exportadoras de aguacate a Estados Unidos de América (tesis doctoral), Instituto Politécnico Nacional, México.

Carmines, E. y R. Zeller (1988), "Reliability and validity assessment, Series Quantitative Applications in the social Sciences", Sage Publications, vol. 17, Beverly Hills, EUA.

Chauca, P. (2000), Administración estratégica para la competitividad de la micro, pequeña y mediana empresa. El caso de las empresas manufactureras de la ciudad de Morelia, Michoacán (tesis doctoral), Instituto Politécnico Nacional, México.

Dickson, P. y J. GinTer (1987), "Market Segmentation, Product Differentiation and Marketing Strategy", Journal of Marketing, 51(4), abril, 1.10, EUA.

Diez de CASTro, E. et al. (2001), Administración y dirección, Mc Graw Hill, Madrid.

Gaither, N. y G. Frazier (2000) Administración de producción y operaciones, 4a. ed., Thompson Editores, México.

Gitman, L. (1986), Fundamentos de administración financiera, 3a. ed., Harla, México.

Guilford, J.P. (1954), Psychometric Methods, McGraw Hill, Nueva York.

Harrison, J. y C. John (2002), Fundamentos de la dirección estratégica, 2a. ed., Thompson Editores, Madrid (Colección Negocios).

Hernández, R. et al. (2003), Metodología de la investigación, 3a. ed., McGraw Hill, México. 
Hill, C. y G. Jones (1996), Administración estratégica, Un enfoque integrado, McGraw Hill, México.

Hofer, C. y D. Schendel (1978), Strategy Formulation: Analytical Concepts, West Publishing, St. Paul. MN, West.

Hodge, B., W. Anthony y L. Gales (1998), Teoría de la organización. Un enfoque estratégico, Prentice Hall, México.

Jefrerey, H. y J. Caron (2002), Fundamentos de dirección estratégica, 2a. edición, Thompson Editores, Madrid.

Kotter, J. y L. Schlesinger (1979), “Choosing Strategies for Change”, Harvard Business Review, vol. 57, núm. 2, Tampa FL.

Likert, R. (1976), "Una técnica para la medición de actitudes", en G. Summers. (comp.), Medición de actitudes, Trillas, México.

Mendoza, N. A. (1998), Manual para determinar necesidades de capacitación y desarrollo, 4a. ed., Trillas, México.

MintzBerg, H. (1988), Generic Strategies: Toward a Comprehensive Framework Advances in Strategic Management, vol. 5, JAI press, Greenwich, ст.

PADUA, J. et al. (1996), Técnicas de investigación aplicadas a las ciencias sociales, El Colegio de México/Fondo de Cultura Económica, México.

Perea, J. (2003) "Estrategias competitivas para los productores cafetaleros mexicanos frente al mercado mundial”, en Investigación Administrativa, núm. 93, año 32, julio-diciembre, ESCA-IPN, México.

Porter, M. (1982), Estrategia competitiva. Técnica para el análisis de los sectores industriales y de la competencia, CECSA, México.

Sallenave, J. P. (1994), La gerencia integral, Grupo Editorial Norma, Bogotá.

SAlminis, J. (2003), "Apuntes sobre la génesis y desarrollo del cooperativismo agropecuario en la república de Argentina”, en REVESCO, Revista de Estudios Cooperativos, núm. 81, III cuatrimestre, Facultad de Ciencias Económicas y Empresariales, Universidad Complutense de Madrid.

Samuelson P. y W. Nordhaus (2006), Economía, 18a. ed., McGraw Hill, México.

Schroeder, R. (1992), Administración de operaciones, 3a. ed., McGraw Hill, México. 
Estrategias competitivas para los productores cafetaleros

de la región de Córdoba, Veracruz, frente al mercado mundial

Stanton, W., M. Etzel y B. Walker (1996), Fundamentos de marketing, 10a. ed., McGraw Hill, México.

Thompson A. y J. Strickland Gamble (2005), Crafing and Executing Strategy. The Quest for Competitive Advantage, Concepts \& Cases, 14a. ed., McGraw Hill Irwin, Nueva York.

Uruchurtu, G. (2004), ¿Cómo Ves?, revista de Divulgación de la Ciencia de la unam, No. 69, año 6, agosto, México.

Wiersma, W. (1999), Research Methods in Education: An introduction, 7a. ed., Allynand Bacon, Boston.

(A) 